Portland State University

PDXScholar

\title{
Continuous and Symmetrical Transformations in the Framework of Classical Particle Systems
}

Nima Laal

Portland State University

Follow this and additional works at: https://pdxscholar.library.pdx.edu/honorstheses

Let us know how access to this document benefits you.

\section{Recommended Citation}

Laal, Nima, "Continuous and Symmetrical Transformations in the Framework of Classical Particle Systems" (2018). University Honors Theses. Paper 597.

https://doi.org/10.15760/honors.607

This Thesis is brought to you for free and open access. It has been accepted for inclusion in University Honors Theses by an authorized administrator of PDXScholar. Please contact us if we can make this document more accessible: pdxscholar@pdx.edu. 
Continuous and Symmetrical Transformations in the Framework of Classical Particle Systems

$$
\text { by }
$$

\title{
Nima Laal
}

An undergraduate honors thesis submitted in partial fulfillment of the

$$
\begin{aligned}
& \text { requirements for the degree of } \\
& \text { Bachelor of Science } \\
& \text { University Honors } \\
& \text { and } \\
& \text { Physics }
\end{aligned}
$$

Thesis Adviser

Professor Pui-Tak Leung

\author{
Portland State University
}




\section{TABLE OF CONTENTS}

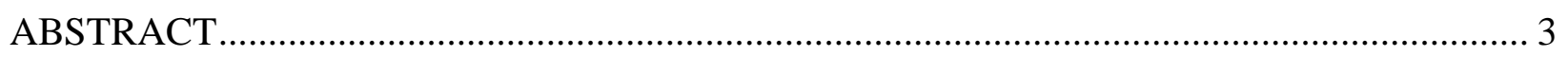

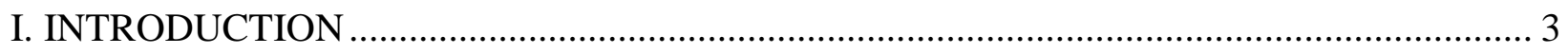

II. THE MEANING OF TRANSFORMATION ............................................................... 4

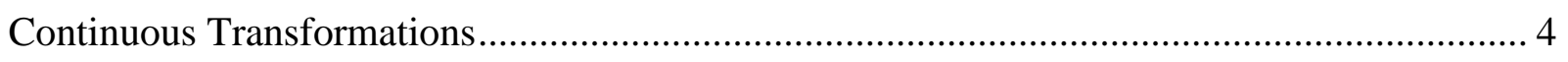

Discrete Transformations .............................................................................................. 5

The Other Types of Transformations.............................................................................. 7

Classifying the Symmetries ........................................................................................ 7

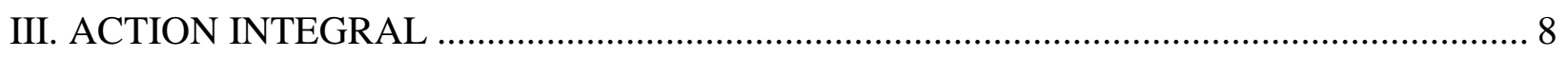

IV. THE ACTION INTEGRAL \& CONTINUOUS SYMMETRIES ..................................... 9

None Relativistic Classical Systems under Continuous Transformations ............................. 12

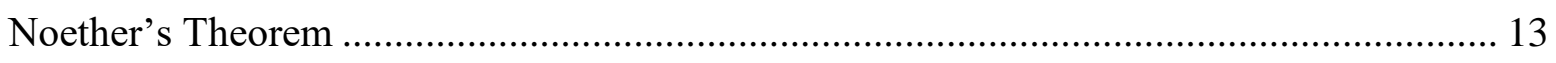

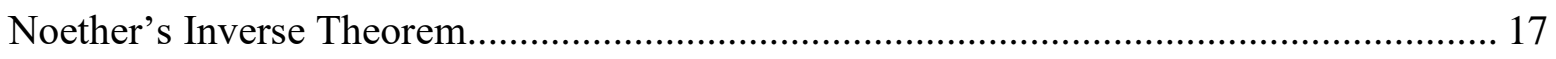

A Note on Noether's Inverse Theorem ......................................................................... 24

V. NOETHER'S THEOREM THROUGH EXAMPLES ..................................................... 24

Example 1 - Noether's Theorem and Conservation of Hamiltonian.................................... 24

Example 2 - Noether's Theorem and Conservation of Conjugate Momentum..................... 27

Example 3 - Noether's Theorem and the Transformation of all Coordinates ........................ 29

Example 4 - The 1-D Conservative Harmonic Oscillator .................................................. 30

Example 5 - An Odd Case: The Lane-Emden Equation ................................................... 32

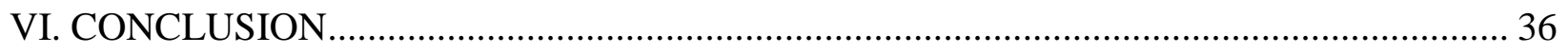

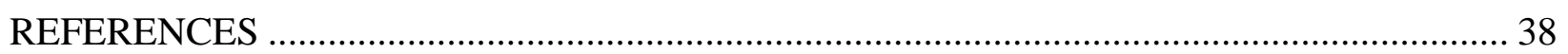




\begin{abstract}
Historically, symmetry has been associated with beauty and perfection, something that is merely a visual characteristic. However, in the realm of physics, symmetry is fundamental: it exists in physical systems and even in the laws of physics. In this study, only one particular type of symmetry - the continuous symmetry- is discussed in great depth. While a discussion of why symmetry exists is not the concern of this paper, the connection that exists between continuous symmetries and conservation laws, through Noether's theorem, is the focus of this study. Despite many articles and books that seek to understand Noether's theorem using Group Theory and Lie symmetry, this study explores the Noether's theorem (and Noether's inverse theorem) for nonrelativistic classical systems using ordinary calculus and an understanding of the concept of continuous transformations.
\end{abstract}

\title{
I. INTRODUCTION
}

Ancient civilizations knew there was something especial about symmetrical characteristics of Nature. They knew that Nature did not have to be symmetrical; yet, it was. No matter where they looked at, they could see some sort of symmetry; any natural system or object was symmetrical in some way. Ancient people believed symmetry to be more than just a characteristic to make objects look more visually appealing; they believed in symmetry as a fundamental principle of Nature, something divine.

Symmetry is in fact more than a visual characteristic. Symmetry exists in Nature, not only in the appearance of objects, but also in the very fundamentals of how objects behave. Physics, as the study of such fundamentals, demonstrates this notion of symmetry elegantly. Physics demonstrates that Nature exhibits symmetry, and there is a significance to this existence.

The motivation for writing this independent study came from this very idea. At first, it seemed silly to think that the notion of symmetry could even be relevant to physics and physical laws. However, after researching about the topic of symmetry in physics, I came to the realization that not only the notion of symmetry, if defined precisely and carefully, is relevant to the study of physical laws, but also could grant a new perspective on how one might think about the physics itself. In this study, I shall investigate one way that the notion of symmetry can enter the realm of physics (that way being the invariance of various systems under continuous transformations), and also observe what consequences this existence has on various physical systems.

Avoiding the artistic meanings of symmetry, the only useful and objective definition for symmetry could be the condition of being invariance under transformations [1]. In other words, if a physical system remains unchanged after a certain occurrence (or more accurately, after a certain transformation), the system is said to exhibit symmetry. This precise definition is the quintessential part of all the rigorous mathematical formulations that will be presented in this study. Although only one class of symmetries, continuous symmetries, will be explored in this paper, the resulting observations and implications of such exploration will be sufficient to prove that the notion of symmetry is deeper and much more complex than our intuition-based understanding of it that is based on regarding symmetry as merely a visual, and sometimes even a trivial, characteristic. 


\section{THE MEANING OF TRANSFORMATION}

There is something very vague about the definition that was presented about the symmetry earlier, particularly about the notion of transformation. It was mentioned earlier that transformation could be thought of as, simply, some type of occurrence or change. Although this is true, this notion is not specific enough to be as of any use: there is an infinite number of ways a system may experience a change. Thus, in order to gain a better understanding of the meaning of transformation, at first, one needs to classify these infinite number of transformations that could occur on a system into finite groups. In this regard, the transformations can be classified into three groups based on their type. Table 1 presents such classification.

\section{TABLE I}

Classification of Types of Transformations [2, chap. 52, "Symmetry in Physical Laws"]

\begin{tabular}{cc}
\hline \hline Types of Transformation & Important Examples \\
1. Continuous & Transformation in Time \\
& Transformation in Space \\
& Transformation in Orientation \\
& Lorentz Transformation \\
2. Discrete & General Reflective Transformation \\
& Reversal of Time \\
& Charge conjugation \\
& Parity Transformation \\
3. Other (Unitary \& Permutation) & Quantum Mechanical Phase Change \\
& Interchange of Identical Particles \\
& Gauge Transformation \\
\hline \hline
\end{tabular}

In this study, only the continuous transformations and their relation to various symmetries are going to be discussed in detail. Nevertheless, I shall briefly explain the characteristics of each of the transformations that are labeled as "important examples" in Table I.

\section{Continuous Transformations}

As the name suggests, continuous transformations are a class of transformations in which transformations continuously change the state of a system. For example, in classical mechanics where time, position, and velocity adequately define the state of a system, a continuous transformation could transform the position of a system continuously, by an infinitesimal amount, for a certain period of time; this is the same as a system moving in space. In section IV, this type of transformation is going to be studied in detail.

The reason that the transformations and their classes are mentioned in this study is because of their connection to the symmetries of physical systems. In the case of continuous 
transformations, the symmetries associated with such transformations are symmetries of time, space, spacial orientation, and perhaps the most important of all, the symmetries of physical laws themselves.

In short, the symmetry of time, space, and spacial orientation means that Nature does not have a preference for location, time, or the orientation. If a certain apparatus is made to do something, for example a mass-spring system undergoing oscillations, then it does not matter where, when, and in which direction the apparatus is doing its job, the dynamics is the same (of course, given all other factors that might affect the performance of the apparatus, such as the damping coefficient in the case of mass-spring system, are also remained unchanged). Now, if the connection of the mentioned symmetries to the continuous transformations were to be explored, something remarkable could be concluded: the symmetries of time, space, and orientation lead to conservation of energy, linear momentum, and angular momentum respectively. In other words, conservation laws could be thought as the consequence of continuous symmetries. This is quite remarkable!

There is one more example of continuous transformation that its connection to symmetries is even more remarkable than the other previously mentioned transformations; this transformation is called the Lorentz transformation. Lorentz transformation is best understood through thought experiments and in the language of mathematics; however, this type of transformation enables us to see the symmetries of physical laws themselves. The physical laws are the same in any inertial frame of reference $^{1}$. In other words, the physics, itself, is symmetrical! As a consequence of this symmetry, no one feels anything different in how everything works when they are riding a car and moving with a constant velocity.

\section{Discrete Transformations}

Discrete transformations are more abstract yet are as important as the continuous transformations. Discrete transformations make sudden and dramatic changes to the state of a system without letting the system to exist in any state in between. For example, the image of an object is obtained by transforming every part of the object through either a point, an axis, or a plane. After the transformation is done, there are only two things: the object and its image, nothing less nothing more. The examples of discrete transformations are the general reflective transformations, time reversal, charge conjugate, and parity transformation.

The example given above about an object and its image illustrates a type of discrete transformation known as the general reflective transformation. The important symmetries associated with this type of transformation are all geometrical in nature. In physics, the quantities can be either scalar or vector; the reflective transformation concerns the vector quantities. When a vector undergoes a reflective transformation, its magnitude remains the same but its direction might change depending on how it was transformed. Thus, the magnitude of any vector is symmetrical under a reflective transformation, but its direction may not. More specifically there

\footnotetext{
${ }^{1}$ The statement the physical laws are the same in any inertial frame of reference is known as the first postulate of the theory of special relativity. In fact, it was this postulate that drew physicists' attention to the concept of symmetry in modern physics [2, chap. 52, "Symmetry in Physical Laws"].
} 
are two types of vectors, polar vectors and axial vectors ${ }^{2}$ (also known as pseudovectors). Only the latter exhibits symmetry, which occurs in the especial case of inversion of all of its coordinate axes. An example of a polar vector is the position vector, and an example of an axial vector is the angular momentum.
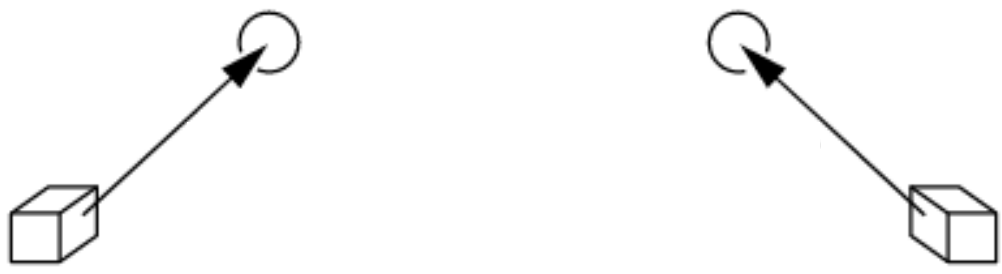

Fig. 1. A polar vector and its image [2, chap. 52, "Symmetry in Physical Laws"]
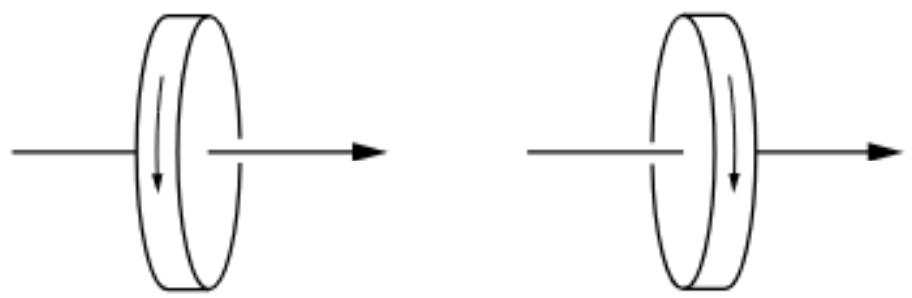

Fig. 2. An axial vector and its image [2, chap. 52, "Symmetry in Physical Laws"]

The other examples of discrete transformations involve the transformation of parity $(\mathrm{P})^{3}$, reversal of time $(\mathrm{T})$, and charge conjugate $(\mathrm{C})$. The details and meanings of these transformations require an understanding of Quantum Mechanics which is not the concern of this paper. For an introductory discussion of these transformation, refer to [2, chap. 52, "Symmetry in Physical Laws"].

The symmetry associated with the CPT transformations is simply called CPT symmetry. According to this symmetry, "if one were to take any lump of matter, reverse the sign of all the elementary charges (C) [the $\mathrm{C}$ transformation is the transformation of particle to its antiparticle], the direction of time's flow (T), and another property of particles called parity (P), the specimen would obey the same laws of physics. CPT invariance is a very general consequence of quantum theory and the covariance of quantum laws under Lorentz transformation demanded by special relativity and is the cornerstone for every modern theory of matter. If CPT invariance is violated, the whole of physical theory at the fundamental level will have to be rewritten" [4].

\footnotetext{
2 Definition: "a polar vector is a vector that reverses sign when the coordinate axes are reversed" [3]. On the other hand, an axial vector is a vector that does not reverse its sign when its coordinate axes are reversed [3].

${ }^{3}$ Parity transformation is very similar to reflective transformation. However, in a reflective transformation, not all the spacial coordinates need to be reversed; whereas all the spacial coordinates reverse their algebraic sign in a parity transformation.
} 


\section{The Other Types of Transformations ${ }^{4}$}

Now, there are only three important transformations left, none of which belong to any other category. Despite their differences, all the three transformations reveal something about the fundamental symmetries of particles and fields. The meaning of transformation in this category is more about the choice between different possibilities rather than an action or a change being done on a system or on a particle. One example of transformation in this class is the exchange of identical particles. The idea is that there is nothing special about any particular electron or water molecule or any other pure entity. All the electrons in the existence are identical as well as all the protons, all the water molecules, etc. Thus, the symmetry in here is the fact that if a particle is exchanged with another particle with the same name, there would not be any difference [2].

Another type of transformation in this class is the quantum mechanical phase change. In quantum mechanics, all the possible information that can be extracted from a system or a quantum mechanical process is imagined to be stored in a mathematical function called wavefunction. This function is expressed in the language of complex variables where there always exists a phase associated with any function. In quantum mechanics, if there are two wavefunctions with the same magnitude but only different phases, the probability that the process expressed by the wavefunctions would occur never changes no matter what the difference in phase is. Physical laws are such that a shift in the quantum-mechanical phase makes no difference. The symmetry associated with this type of transformation leads to the conservation of probability.

There is yet another type of transformation in this class. This transformation is called gauge transformation. The essence of gauge transformation is that "the choice of non-observable properties of fields (e.g. potentials) does not affect the observable properties of fields, such as the intensity of electric or magnetic fields" [5]. The symmetry associated with this type of transformation is known as gauge symmetry, and the conservation of electrical charge is an important consequence of this symmetry.

\section{Classifying the Symmetries}

Using the information presented above, along with some extra information ${ }^{5}$, one can make a classification on various symmetries of systems. Figure 3 presents such classification. In this paper, only the continuous symmetries (in the framework of classical particle dynamics) will be explored.

\footnotetext{
${ }^{4}$ This class of transformation is the subject of study of quantum mechanics.

5 This "extra information" is Noether's theorem which will be discussed in detail in this paper.
} 


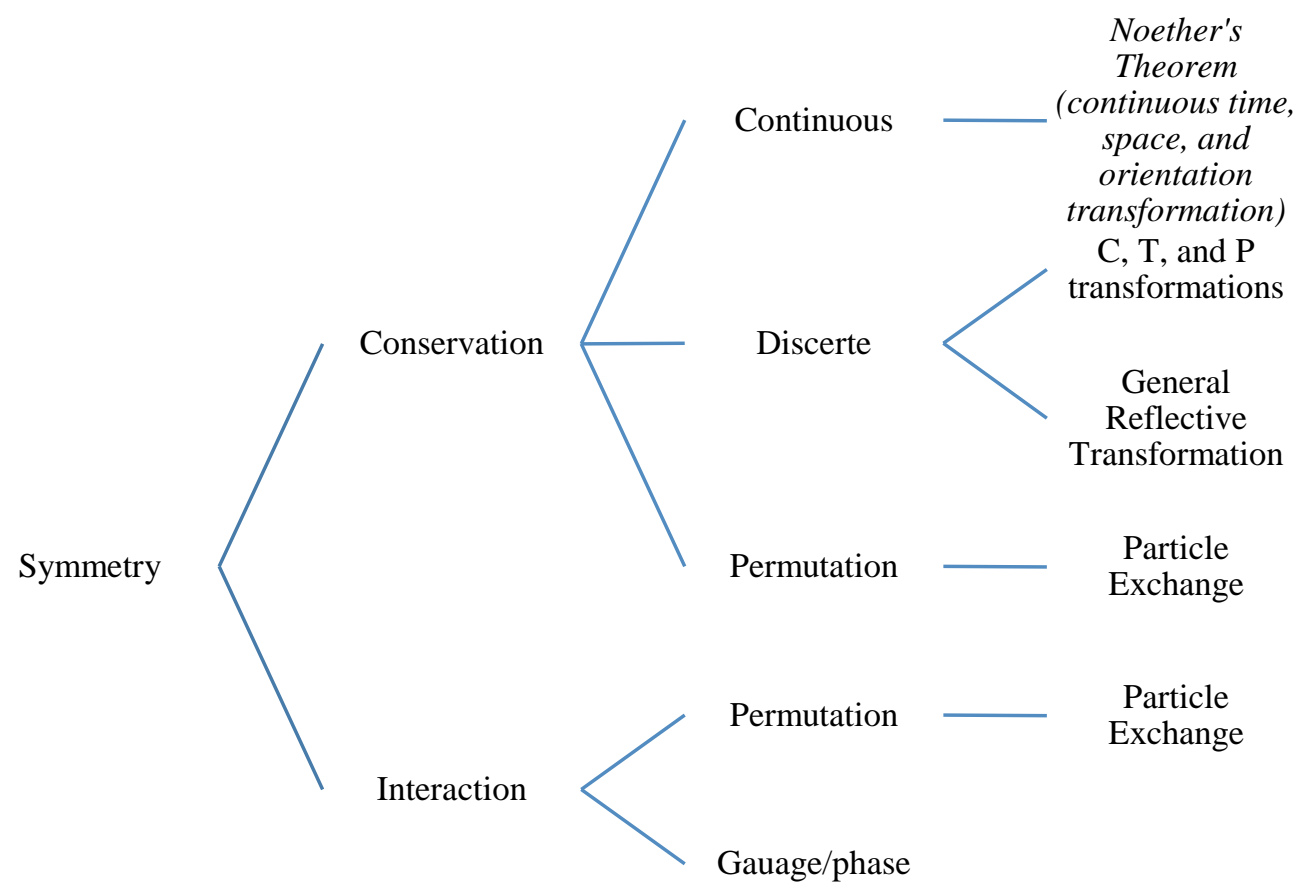

Fig. 3. Classification of types of symmetries in physics.

\section{ACTION INTEGRAL}

Our intuition about the world we live in has been built based on our experience with how Nature behaves. Our intuition tells us that if a ball is thrown straight upward, it will eventually fall down, or if a rock is thrown with an angle above the ground, it will follow a curved path and hit the ground at the end. But why is all of that true? Why every time we throw an object in a certain direction, we expect it to behave in a certain way; and it never fails to meet our expectation? The Principle of Least Action (also known as Hamilton's Principle) is the closest that we have ever been to answer these simple yet deep questions.

According to the principle of least action, although there is an infinite number of ways a system may behave, it only chooses to behave in one particular way over and over again because that particular way is the way that extremizes, what physicists call, the action integral ${ }^{6}$, denoted by $S$ and defined explicitly as:

$$
S=\int_{t_{1}}^{t_{2}} L\left(x^{i}, \dot{x}^{i}, t\right) d t
$$

\footnotetext{
${ }^{6}$ Extremizing the action integral refers to either minimizing the action integral or making it a saddle point. More discussion of this concept, including the reason why the action integral cannot be maximized, can be found at [6].
} 
where $i \in\{1,2,3\}$ such that $x^{1} \equiv x, x^{2} \equiv y$, and $x^{3} \equiv z$. Ignoring the intimidating look of the above equation, all it says is that the action integral is a unique number for each of the possible paths a system may undergo (i.e. a number assigned to every possible behavior of a system). For example, in the case of throwing a ball upward, the Action integral might be calculated to be 5 if it follows the observed and familiar path of rising and falling while moving straight, or action integral might be calculated to be 8 if it follows an unexpected path of rising and falling while moving in a zig-zag. Nonetheless, given that the physical system of which the Lagrangian is assigned to is under a potential energy $U$ where

$$
\frac{\partial^{2} U}{\partial x^{2}} \leq 0
$$

along the entire worldline ${ }^{7}$, which is always the case for throwing a ball while being on earth, the observed behavior is ALWAYS the path with the smallest number according to the principle of least action [6].

The function $L\left(x^{i}, \dot{x}^{i}, t\right)$ is called the Lagrangian, and it is the difference between the kinetic and potential energy of a classical non-relativistic system at any moment in time. In a sense, the principle of least action states that the observed and true behavior of a system is the one in which this difference between the kinetic and potential energy is minimized (given a short worldline or satisfaction of equation III.2 [6]). The further details ${ }^{8}$ of the Lagrangian and action integral are not particularly important for the purpose of this paper; however, the consequence of the principle of least action in resulting in a relationship between different physical quantities of a system is as of great importance to the goal of this paper. This relationship is known as the Euler-Lagrange equation:

$$
\text { For Particles : } \frac{\partial L}{\partial x^{i}}=\frac{d}{d t}\left(\frac{\partial L}{\partial \dot{x}^{i}}\right)
$$

Where, again, $i$ belongs to the set $\{1,2,3\}$ where $x^{1}=x, x^{2}=y$, and $x^{3}=z$. Once the principle of least action and its most important consequence (the Euler-Lagrange equation) is understood, we have enough tools in our disposal to begin the mathematical discussion of symmetry.

\section{THE ACTION INTEGRAL \& CONTINUOUS SYMMETRIES}

The principle of least action is only capable of determining how a single well-defined system (a system with a known kinetic and potential energy in a classical non-relativistic framework) behaves. But, what happens if the system undergoes changes and transformations? What are the consequences of transformations on the behavior of a previously well-understood

\footnotetext{
${ }^{7}$ Please refer to [6] for more discussion on this topic.

${ }^{8}$ Additional details on this topic can be found at [2].
} 
system? With the help of our precise definition of symmetry that is the condition of being invariant under transformations and also rigorous mathematical manipulation, we can adequately address these questions.

In the presented definition of symmetry, there are only two key terms: first key term being invariance, and the second key term being transformation. We first need to define each key term as precisely as possible in order to proceed further with the consequences of the existence of symmetry. As was previously mentioned, continuous symmetries are the subject of study of this paper; hence, the definition of invariance needs to be fully compatible with the nature of continuous transformations. In order to have a useful definition of invariance for continuous transformations, it is helpful to see what happens to the action integral $S$ when the Lagrangian undergoes a general continuous transformation.

Given an action

$$
S=\int_{t_{1}}^{t_{2}} L\left(x^{i}, \dot{x}^{i}, t\right) d t
$$

for $i=1,2,3$, the act of transforming the coordinates would result in another action, expressed by

$$
\begin{aligned}
S^{\prime} & =\int_{t^{\prime}{ }_{1}}^{t^{\prime}} L\left(x^{\prime}, \dot{x}^{\prime}, t^{\prime}\right) d t^{\prime} \\
& =\int_{t^{\prime}{ }_{1}}^{t^{\prime} 2} L^{\prime} d t^{\prime} .
\end{aligned}
$$

Furthermore, based on the most general form of a continuous coordinate transformation, expressed explicitly by

$$
\left\{\begin{array}{c}
t \rightarrow t^{\prime}=t+\delta t=t+\varepsilon \tau\left(t, x^{i}\right) \\
x^{i} \rightarrow x^{i}=x^{i}+\delta x^{i}=x^{i}+\varepsilon \lambda^{i}\left(x^{j}, \mathrm{t}\right)
\end{array}\right\}
$$

where $\tau$ and $\lambda^{i}$ are functions of position and time and $\varepsilon$ is a constant that is infinitesimally small [7], [8], [9], the change of action from $S$ to $S$ ' can be calculated as follows:

$$
\begin{aligned}
S^{\prime}-S & =\int_{t_{1}^{\prime}}^{t^{\prime}} L^{\prime} d t^{\prime}-\int_{t_{1}}^{t_{2}} L d t \\
& =\int_{t_{1}}^{t_{2}}\left[L^{\prime} \frac{d t^{\prime}}{d t}-L\right] d t \\
& =\int_{t_{1}}^{t_{2}}\left[L^{\prime}\left(1+\frac{d}{d t} \delta t\right)-L\right] d t
\end{aligned}
$$

in which a $U$-substitution was performed to put the two integrals inside one single integral with one single limit of integration. Hence,

$$
\therefore S^{\prime}-S=\int_{t_{1}}^{t_{2}}\left[\left(L^{\prime}-L\right)+L^{\prime} \frac{d(\delta t)}{d t}\right] d t .
$$


It is a consequence of action principle that for a given system, one can write an infinite number of Lagrangians equally valid and all resulting in the same equation(s) of motion. These Lagrangians are different from each other by a total time derivative term

$$
\frac{d g\left(x^{i}, t\right)}{d t}
$$

where $g$ cannot depend explicitly on velocity and it should have identical values at boundaries that is

$$
g\left(x^{i}\left(t_{1}\right), t_{1}\right)=g\left(x^{i}\left(t_{2}\right), t_{2}\right)
$$

(otherwise, the equation(s) of motion will be altered by a change of Lagrangian). Thus, $L^{\prime}-L$ term of equation IV.4 can be replaced by $d g\left(x^{i}, t\right) / d t$. Furthermore, the remaining term inside the bracket in equation IV.4 can be rewritten as a total time derivative term, expressed explicitly as

$$
\frac{d}{d t} h\left(x^{i}, t\right) \equiv L^{\prime} \frac{d(\delta t)}{d t}
$$

Similar to $g, h$ cannot depend on velocity, but for a different reason. If $h$ were to depend on velocity, this would suggest an acceleration dependence term to exist in the product of $L^{\prime}$ and $d \delta t / d t$ which is prohibited by the action principle as well as the definition of the continuous coordinate transformation given in IV.3.

Thus, knowing more about each term of equation IV.4, this equation can be rewritten in a more compact form:

$$
\begin{gathered}
S^{\prime}-S=\int_{t_{1}}^{t_{2}}\left[\left(\frac{d}{d t} g\left(x^{i}, t\right)\right)+\frac{d}{d t} h\left(x^{i}, t\right)\right] d t \\
=\int_{t_{1}}^{t_{2}}\left[\frac{d}{d t}\left(g\left(x^{i}, t\right)+h\left(x^{i}, t\right)\right)\right] d t \\
\therefore S^{\prime}-S=\int_{t_{1}}^{t_{2}}\left[\frac{d}{d t}\left(K\left(x^{i}, t\right)\right)\right] d t .
\end{gathered}
$$

where $K$ is a function of $x^{i}$ and $t$. Thus, based on the above derivation, if the invariance of $S$ is desired, the mathematical form of the invariance could be expressed as

$$
\delta S=\int\left[\frac{d}{d t}\left(K\left(x^{i}, t\right)\right)\right] d t .
$$

I shall take this as the definition of invariance [7] [8].

Now that the definition of invariance is well understood for the continuous transformations, we can start the formal discussion of symmetry. This discussion concerns the non-relativistic classical systems under continuous transformations. 


\section{None Relativistic Classical Systems under Continuous Transformations}

The definition of Invariance for this case, as justified previously, is:

$$
\delta S \equiv \int_{t_{1}^{\prime}}^{t^{\prime}} L\left(x^{i}, \dot{x}^{i}, t^{\prime}\right) d t^{\prime}-\int_{t_{1}}^{t_{2}} L\left(x^{i}, \dot{x}^{i}, t\right) d t=\int_{t_{1}}^{t_{2}} \frac{d}{d t}\left(K\left(x^{i}, t\right)\right) d t .
$$

Moreover, the types of transformations considered for this case are

$$
\begin{aligned}
& t \rightarrow t^{\prime}=t+\delta t \\
& x^{i} \rightarrow x^{\prime i}=x^{i}+\delta x^{i} \\
& \dot{x}^{i} \rightarrow x^{\prime i}=\frac{d}{d t^{\prime}}\left(x^{\prime} i\right)
\end{aligned}
$$

where the prim sign indicates a transformed quantity and $\delta$ indicates an infinitesimal change. Evaluating the velocity transformation further suggests

$$
\begin{aligned}
\dot{x}^{\prime i} & =\frac{d}{d t^{\prime}}\left(x^{\prime i}\right) \\
& =\frac{d x^{i}+d\left(\delta x^{i}\right)}{d t+d(\delta t)} \\
& =\frac{\dot{x}^{i}+\frac{d}{d t}\left(\delta x^{i}\right)}{1+\frac{d(\delta t)}{d t}} \\
& \approx \dot{x}^{i}+\frac{d}{d t}\left(\delta x^{i}\right)-\dot{x}^{i} \frac{d}{d t}(\delta t) \\
& =\dot{x}^{i}+\left(\frac{d\left(\delta x^{i}\right)}{d t}-\dot{x}^{i} \frac{d(\delta t)}{d t}\right) \equiv \dot{x}^{i}+\delta v^{i},
\end{aligned}
$$

noting that $\delta v^{i}$ is used instead of $\delta \dot{x}^{i}$ to express the change in $\dot{x}^{i}$ in order to avoid a potential confusion that may occur since $\delta \dot{x}^{i}$ means $d\left(\delta x^{i}\right) / d t$ whereas the change in $\dot{x}^{i}$ is not necessarily $d\left(\delta x^{i}\right) / d t$ as shown above. Therefore, the following is the complete set of continuous transformations:

$$
\begin{aligned}
& t \rightarrow t^{\prime}=t+\delta t \\
& x^{i} \rightarrow x^{\prime i}=x^{i}+\delta x^{i} \\
& \dot{x}^{i} \rightarrow x^{\prime i}=\dot{x}^{i}+\delta v^{i} \quad ; \delta v^{i} \equiv\left(\frac{d\left(\delta x^{i}\right)}{d t}-\dot{x}^{i} \frac{d(\delta t)}{d t}\right)
\end{aligned}
$$


It is worth mentioning that as a premise of this set of continuous transformations, the spacial and time transformations are considered independent of each other. Thus, although a transformation in time could result in a change in any of the $x^{i}$ coordinates (i.e. $x^{i}(t) \rightarrow x^{\prime} i(t)=x^{i}(t+\delta t)$ ), that change is not the type of change I regard as a spacial transformation since time and spacial transformations have to be independent of each other.

The following section derives what is known as Noether's theorem. First proven by German mathematician Emmy Noether in 1915 [7], this theorem does exactly what we want: revealing the consequences of existence of symmetry ${ }^{9}$.

\section{Noether's Theorem ${ }^{10}$}

From the set of transformations of IV.9,

$$
\frac{d t^{\prime}}{d t}=1+\frac{d(\delta t)}{d t}
$$

Therefore,

$$
\int L\left(x^{\prime}, \dot{x}^{\prime}, t^{\prime}\right) d t^{\prime}=\int\left[L\left(x^{\prime} i, \dot{x}^{\prime}, t^{\prime}\right)\left(1+\frac{d(\delta t)}{d t}\right)\right] d t
$$

resulting in

$$
\delta S=\int\left[\left(L^{\prime}-L\right)+L^{\prime}\left(\frac{d(\delta t)}{d t}\right)\right] d t
$$

where $L\left(x^{\prime}, \dot{x}^{\prime}, t^{\prime}\right) \equiv L^{\prime}$.

For any function $f(x, y, z)$, and for $\delta x, \delta y, \delta z<<1$,

$$
f(x+\delta x, y+\delta y, z+\delta z) \simeq f(x, y, z)+\frac{\partial f}{\partial x} \delta x+\frac{\partial f}{\partial y} \delta y+\frac{\partial f}{\partial z} \delta z
$$

up to the first order approximation. Thus:

$$
L^{\prime}=L+\frac{\partial L}{\partial t} \delta t+\frac{\partial L}{\partial x^{i}} \delta x^{i}+\frac{\partial L}{\partial \dot{x}^{i}} \delta v^{i}
$$

\footnotetext{
${ }^{9}$ One might argue that Noether's theorem only demonstrates how symmetries are connected to conservation laws rather than how symmetries might be the reason for existence of conservation laws. At the end of this study, it is up to the reader to decide which statement is true.

10 The derivation of Noether's theorem presented here is inspired by [8], but, it is a more general version of the derivation presented in [8].
} 
Putting IV.14 into IV.12, results in

$$
\begin{aligned}
\delta S & =\int\left[\left(L+\frac{\partial L}{\partial t} \delta t+\frac{\partial L}{\partial x^{i}} \delta x^{i}+\frac{\partial L}{\partial \dot{x}^{\mu}} \delta v^{i}-L\right)+L^{\prime}\left(\frac{d(\delta t)}{d t}\right)\right] d t \\
& =\int\left[\left(\frac{\partial L}{\partial t} \delta t+\frac{\partial L}{\partial x^{i}} \delta x^{i}+\frac{\partial L}{\partial \dot{x}^{i}} \delta v^{i}\right)+L^{\prime}\left(\frac{d(\delta t)}{d t}\right)\right] d t .
\end{aligned}
$$

From the method of integration by parts, for any two well-behaved functions $U$ and $V$

$$
U V-\int U d V=\int V d U
$$

Let $U=\frac{\partial L}{\partial \dot{x}^{i}}$ and $d V=\delta v^{i} d t$. Therefore, using the definition of $\delta v^{i}$ given in IV.9,

$$
\begin{gathered}
\int \frac{\partial L}{\partial \dot{x}^{i}} \delta v^{i} d t=\frac{\partial L}{\partial \dot{x}^{i}}\left(\delta x^{i}-\int\left(\dot{x}^{i} \frac{d(\delta t)}{d t}\right) d t\right)-\int\left[\left(\delta x^{i}-\int\left(\dot{x}^{i} \frac{d(\delta t)}{d t}\right) d t\right) \frac{d}{d t}\left(\frac{\partial L}{\partial \dot{x}^{i}}\right)\right] d t \\
\int \frac{\partial L}{\partial \dot{x}^{i}} \delta v^{i} d t=\frac{\partial L}{\partial \dot{x}^{i}}\left(\delta x^{i}-E\left(\dot{x}^{i}, x^{i}, t\right)\right)-\int\left[\left(\delta x^{i}-E\left(\dot{x}^{i}, x^{i}, t\right)\right) \frac{d}{d t}\left(\frac{\partial L}{\partial \dot{x}^{i}}\right)\right] d t,
\end{gathered}
$$

for

$$
E\left(\dot{x}^{i}, x^{i}, t\right) \equiv E \equiv \int\left(\dot{x}^{i} \frac{d(\delta t)}{d t}\right) d t .
$$

Putting IV.16 into IV.15 results in

$$
\delta S=\int\left[\left(\frac{\partial L}{\partial x^{i}}-\frac{d}{d t}\left(\frac{\partial L}{\partial \dot{x}^{i}}\right)\right) \delta x^{i}\right] d t+\int\left[\frac{\partial L}{\partial t} \delta t+\frac{d}{d t}\left(\frac{\partial L}{\partial \dot{x}^{i}}\right)(E)+L^{\prime}\left(\frac{d(\delta t)}{d t}\right)\right] d t+\frac{\partial L}{\partial \dot{x}^{i}}\left(\delta x^{i}-E\right) .
$$

By Euler-Lagrange equation, the first expression inside the bracket is zero. Thus,

$$
\begin{aligned}
\delta S & =\int\left[\frac{\partial L}{\partial t} \delta t+\frac{d}{d t}\left(\frac{\partial L}{\partial \dot{x}^{i}}\right) E+L^{\prime}\left(\frac{d(\delta t)}{d t}\right)\right] d t+\frac{\partial L}{\partial \dot{x}^{i}}\left(\delta x^{i}-E\right) \\
& =\int\left[\frac{\partial L}{\partial t} \delta t+\frac{d}{d t}\left(\frac{\partial L}{\partial \dot{x}^{i}}\right) E+L^{\prime}\left(\frac{d(\delta t)}{d t}\right)\right] d t+\int\left[\frac{d}{d t}\left(\frac{\partial L}{\partial \dot{x}^{i}}\left(\delta x^{i}-E\right)\right] d t\right. \\
& =\int\left[\left(\frac{\partial L}{\partial t} \delta t+\frac{d}{d t}\left(\frac{\partial L}{\partial \dot{x}^{i}} \delta x^{i}\right)\right)+\frac{d}{d t}\left(\frac{\partial L}{\partial \dot{x}^{i}}\right) E-\left(\frac{d}{d t}\left(\frac{\partial L}{\partial \dot{x}^{i}} E\right)\right)+L^{\prime}\left(\frac{d(\delta t)}{d t}\right)\right] d t \\
& =\int\left[\left(-\dot{H} \delta t+\frac{d}{d t}\left(p^{i} \delta x^{i}\right)\right)+\left(\dot{p}^{i} E\right)-\left(\dot{p}^{i} E+p^{i} \dot{E}\right)+L^{\prime}\left(\frac{d(\delta t)}{d t}\right)\right] d t .
\end{aligned}
$$




$$
\therefore \delta S=\int\left[\left(-\dot{H} \delta t+\frac{d}{d t}\left(p^{i} \delta x^{i}\right)\right)-p^{i} \dot{E}+L^{\prime}\left(\frac{d(\delta t)}{d t}\right)\right] d t \text {, }
$$

where $H$ is the Hamiltonian and $p^{i}$ is the momentum conjugate to $x^{i}$.

In order to proceed further with equation IV.17, one has to determine whether or not the last term inside the integral of IV.17 can be simplified further. Writing $L^{\prime}$ in terms of $L$ reveals that

$$
\begin{aligned}
L^{\prime} \frac{d(\delta t)}{d t} & =\left(L+\frac{\partial L}{\partial t} \delta t+\frac{\partial L}{\partial x^{i}} \delta x^{i}+\frac{\partial L}{\partial \dot{x}^{i}} \delta \dot{x}\right) \frac{d(\delta t)}{d t} \\
& \approx L \frac{d(\delta t)}{d t},
\end{aligned}
$$

up to the first order approximation. Moreover, based on the definition of Hamiltonian,

$$
H \equiv p^{i} \dot{x}^{i}-L
$$

Putting IV.19 into IV.18 results in

$$
L \frac{d(\delta t)}{d t}=\frac{d(\delta t)}{d t}\left(p^{i} \dot{x}^{i}\right)-\left(H \frac{d(\delta t)}{d t}\right) .
$$

Now, equation IV.20 can be used to evaluate equation IV.17 further:

$$
\begin{aligned}
\delta S= & \int\left[\left(-\dot{H} \delta t+\frac{d}{d t}\left(p^{i} \delta x^{i}\right)\right)-p^{i} \dot{E}+L^{\prime}\left(\frac{d(\delta t)}{d t}\right)\right] d t \\
= & \int\left[\left(-\dot{H} \delta t+\frac{d}{d t}\left(p^{i} \delta x^{i}\right)\right)-p^{i} \dot{E}+\frac{d(\delta t)}{d t}\left(p^{i} \dot{x}^{i}\right)-\left(H \frac{d(\delta t)}{d t}\right)\right] d t \\
& =\int\left[-\frac{d}{d t}(H \delta t)+\frac{d}{d t}\left(p^{i} \delta x^{i}\right)-p^{i} \dot{E}^{i}+\frac{d(\delta t)}{d t}\left(p^{i} \dot{x}^{i}\right)\right] d t
\end{aligned}
$$

Recalling the definition of $E$ which states

$$
E=\int\left(\dot{x}^{i} \frac{d(\delta t)}{d t}\right) d t
$$

it is easy to show that

$$
\dot{E}=\dot{x}^{i} \frac{d(\delta t)}{d t}
$$

which further simplifies equation IV.21 into 


$$
\begin{aligned}
\delta S & =\int\left[-\frac{d}{d t}(H \delta t)+\frac{d}{d t}\left(p^{i} \delta x^{i}\right)-p^{i} \dot{E}^{i}+p^{i} \dot{E}^{i}\right] d t \\
& =\int\left[\frac{d}{d t}\left(-H \delta t+p^{i} \delta x^{i}\right)\right] d t .
\end{aligned}
$$

Furthermore, based on the definition of invariance given in IV.8, the following can be concluded:

$$
\delta S=\int\left[\frac{d}{d t}\left(K\left(x^{i}, t\right)\right)\right] d t=\int\left[\frac{d}{d t}\left(-H \delta t+p^{i} \delta x^{i}\right)\right] d t
$$

Thus, resulting in

$$
\therefore Q=p^{i} \delta x^{i}-H \delta t-K\left(x^{i}, t\right)=\text { Const. }
$$

where $Q$ is Noether's Conserved Charge. The derivation of Noether's theorem for classical systems is now complete. Noether's conserved charge was achieved using the definition of invariance- $\delta S=\int \frac{d}{d t}\left(K\left(x^{i}, t\right)\right) d t$ - and for the general set of continuous transformations defined by IV.9. This equation is referred to as Noether's first theorem (for classical dynamics).

The purely mathematical way of obtaining Noether's conserved charge (equation IV.22) may not convey its true significance. Nevertheless, it is an outstanding and powerful equation. Equation IV.22 states that a conservation law could be obtained if a classical particle system undergoes a continuous transformation in the form of IV.9. The set of transformations presented in IV.9 is the definition of continuous transformations. Noether's theorem only concerns such transformations.

Nevertheless, not all continuous transformations lead to conserved quantities. There is a specific set of transformations in the form suggested by IV.9 along with some additional restrictions that would guarantee a Noether's conserved charge (i.e. a conservation law). These transformations are called symmetrical transformations; transformations that lead to conservation laws. Thus far, only the general form of symmetrical transformations has been discussed.

In order to find the requirements for symmetrical transformations (besides equation IV.9), we need to take advantage of what is known as Noether's inverse theorem. According to this theorem, not all continuous transformations lead to conserved quantities. For a given Lagrangian, there is a set of conditions that continuous transformations (more specifically $\delta t$ and $\delta x^{i}$ ) have to satisfy in order for Noether's formalism to result in a conserved quantity. These conditions are referred to as killing equations.

In what follows, I shall prove Noether's inverse theorem and find the exact conditions that would turn continuous transformations into symmetrical transformations. Once the symmetrical transformations are known explicitly, then we can claim that we have understood everything about the connection between continuous transformations and conserved quantities. 


\section{Noether's Inverse Theorem [9] $]^{11}$}

For a constant $\varepsilon$, where $\varepsilon<<1$, the set of transformations of IV.9 can be rewritten as:

$$
\begin{aligned}
& t \rightarrow t^{\prime}=t+\varepsilon \tau\left(t, x^{i}\right) \\
& x^{i} \rightarrow x^{\prime i}=x^{i}+\varepsilon \lambda\left(t, x^{j}\right) \\
& \dot{x}^{i} \rightarrow \dot{x}^{\prime}=\frac{d\left(x^{\prime}\right)}{d t^{\prime}}
\end{aligned}
$$

where $\tau\left(t, x^{j}\right) \equiv \tau$ and $\lambda^{i} \equiv \lambda^{i}\left(t, x^{j}\right)$ are two continuous functions. Furthermore, the velocity transformation of IV.23 has to be found explicitly:

$$
\begin{gathered}
\dot{x}^{\prime i}=\frac{d\left(x^{\prime i}\right)}{d t^{\prime}}=\frac{d\left(x^{i}+\varepsilon \lambda^{i}\right)}{d t+d(\varepsilon \tau)} \\
\dot{x}^{\prime}=\frac{d x^{i}+\varepsilon d\left(\lambda^{i}\right)}{d t+\varepsilon d(\tau)} \\
=\frac{\dot{x}^{i}+\varepsilon \dot{\lambda}^{i}}{1+\varepsilon \dot{\tau}} \\
=\left(\dot{x}^{i}+\varepsilon \dot{\lambda}^{i}\right)(1+\varepsilon \dot{\tau})^{-1} \\
\approx\left(\dot{x}^{i}+\varepsilon \dot{\lambda}^{i}\right)(1-\varepsilon \dot{\tau}) \\
\approx \dot{x}^{i}+\varepsilon\left(\dot{\lambda}^{i}-\dot{x}^{i} \dot{\tau}\right)
\end{gathered}
$$

up to the first order approximation. Thus, the transformations of IV.23 can be rewritten as

$$
\begin{aligned}
& t \rightarrow t^{\prime}=t+\varepsilon \tau\left(t, x^{i}\right) \\
& x^{i} \rightarrow x^{\prime i}=x^{i}+\varepsilon \lambda^{i}\left(t, x^{j}\right) \\
& \dot{x}^{i} \rightarrow \dot{x}^{i}=\dot{x}^{i}+\varepsilon\left(\dot{\lambda}^{i}-\dot{x}^{i} \dot{\tau}\right) .
\end{aligned}
$$

On one hand, according to Noether's theorem, if there is a conserved charge; it can be written as

$$
Q=p^{i} \delta x^{i}-H \delta t-K\left(x^{i}, t\right)
$$

On the other hand, based on the set of transformations of IV.24,

\footnotetext{
${ }^{11}$ The derivation of Noether's inverse theorem presented here is a more thorough version of the derivation presented
} in [9]. Please refer to [9] for a succinct derivation of Noether's inverse theorem. 


$$
\begin{aligned}
& \delta x^{i}=\varepsilon \lambda^{i} \\
& \delta t=\varepsilon \tau
\end{aligned}
$$

Putting IV.25 into IV.24 results in

$$
\frac{Q}{\varepsilon}=p^{i} \lambda^{i}-H \tau-\frac{K}{\varepsilon}
$$

Since $Q$ is a constant, $Q / \varepsilon$ is a constant as well. In addition, the term $K / \varepsilon$ is merely a rescaled version of $K$. Therefore, if $K / \varepsilon \equiv f\left(t, x^{i}\right)$, then

$$
p^{i} \lambda^{i}-H \tau-f=\text { const. }
$$

which is just a different form of equation IV.22, highlighting the functional forms of spacial and time transformations.

Considering

$$
H=\frac{1}{2 m}\left(p^{i}\right)^{2}+U\left(x^{i}, t\right)
$$

as a typical Hamiltonian of a classical system, equation IV.27 can be rewritten as

$$
p^{i} \lambda^{i}-\left(\frac{1}{2 m} p^{i 2}+U\left(x^{i}, t\right)\right) \tau-f=\text { const } .
$$

Taking the total time derivative of both sides of the above equation results in

$$
\dot{p}^{i} \lambda^{i}+p^{i} \dot{\lambda}^{i}-\tau\left(\frac{\partial U}{\partial t}\right)-\dot{\tau}\left(\frac{1}{2 m} p^{i 2}+U\left(x^{i}, t\right)\right)-\frac{d f}{d t}=0
$$

where $\partial U / \partial t=\dot{H}$. By the chain rule of calculus and the exact expression of conjugate momentum for a classical system with the Hamiltonian presented above,

$$
\begin{aligned}
\dot{\lambda}^{i}\left(t, x^{j}\right) & =\frac{\partial \lambda^{i}}{\partial x^{j}} \dot{x}^{j}+\frac{\partial \lambda^{i}}{\partial t} \\
& =\frac{p^{j}}{m} \frac{\partial \lambda^{i}}{\partial x^{j}}+\frac{\partial \lambda^{i}}{\partial t} \\
\dot{\tau}\left(t, x^{i}\right) & =\frac{\partial \tau}{\partial x^{i}} \dot{x}^{i}+\frac{\partial \tau}{\partial t} \\
& =\frac{p^{i}}{m} \frac{\partial \tau}{\partial x^{i}}+\frac{\partial \tau}{\partial t} \\
\dot{f}\left(t, x^{i}\right) & =\frac{\partial f}{\partial x^{i}} \dot{x}^{i}+\frac{\partial f}{\partial t}
\end{aligned}
$$




$$
=\frac{p^{i}}{m} \frac{\partial f}{\partial x^{i}}+\frac{\partial f}{\partial t} .
$$

Putting these derivative terms back into equation IV.28 results in

$$
\begin{aligned}
0 & =\dot{p}^{i} \lambda^{i}+p^{i}\left(\frac{p^{j}}{m} \frac{\partial \lambda^{i}}{\partial x^{j}}+\frac{\partial \lambda^{i}}{\partial t}\right)-\tau\left(\frac{\partial U}{\partial t}\right)-\left(\frac{p^{i}}{m} \frac{\partial \tau}{\partial x^{i}}+\frac{\partial \tau}{\partial t}\right)\left(\frac{1}{2 m}\left(p^{i}\right)^{2}+U\left(x^{i}, t\right)\right)-\left(\frac{p^{i}}{m} \frac{\partial f}{\partial x^{i}}+\frac{\partial f}{\partial t}\right) \\
& =-\frac{\partial U}{\partial x^{i}} \lambda^{i}+p^{i}\left(\frac{p^{j}}{m} \frac{\partial \lambda^{i}}{\partial x^{j}}+\frac{\partial \lambda^{i}}{\partial t}\right)-\tau\left(\frac{\partial U}{\partial t}\right)-\left(\frac{p^{i}}{m} \frac{\partial \tau}{\partial x^{i}}+\frac{\partial \tau}{\partial t}\right)\left(\frac{1}{2 m}\left(p^{i} p^{j} \delta_{i j}\right)+U\left(x^{i}, t\right)\right) \\
& -\left(\frac{p^{i}}{m} \frac{\partial f}{\partial x^{i}}+\frac{\partial f}{\partial t}\right)
\end{aligned}
$$

where $\delta_{i j}$ is the Kronecker delta for which if $i=j$ then $\delta_{i j}=1$ otherwise $\delta_{i j}=0^{12}$. Continuing the derivation leads to

$$
\begin{aligned}
0 & =p^{i}\left(\frac{\partial \lambda^{i}}{\partial t}-\frac{1}{m} \frac{\partial f}{\partial x^{i}}-\frac{1}{m} \frac{\partial \tau}{\partial x^{i}} U\right)+p^{i} p^{j}\left(\frac{1}{m} \frac{\partial \lambda^{i}}{\partial x^{j}}-\frac{\delta_{i j}}{2 m} \frac{\partial \tau}{\partial t}\right)+\left(p^{i}\right)^{2} p^{j}\left(-\frac{\delta_{i j}}{2 m^{2}} \frac{\partial \tau}{\partial x^{i}}\right) \\
& +\left(-\frac{\partial \tau}{\partial t} U-\tau \frac{\partial U}{\partial t}-\frac{\partial U}{\partial x^{i}} \lambda^{i}-\frac{\partial f}{\partial t}\right) .
\end{aligned}
$$

In order for equation IV.29 to hold for any value of $p^{i}$ and $p^{j}$, each expression inside the parenthesis of equation IV.29 has to vanish. This results in three coupled and one uncoupled equation:

$$
\therefore\left\{\begin{array}{l}
\left(p^{i}\right)^{0}\left[-\frac{\partial \tau}{\partial t} U-\tau \frac{\partial U}{\partial t}-\frac{\partial U}{\partial x^{i}} \lambda^{i}-\frac{\partial f}{\partial t}\right]=0 \\
\left(p^{i}\right)^{1}\left[\frac{\partial \lambda^{i}}{\partial t}-\frac{1}{m} \frac{\partial f}{\partial x^{i}}-\frac{1}{m} \frac{\partial \tau}{\partial x^{i}} U\right]=0 \\
\left(p^{i} p^{j}\right)\left[\frac{1}{m} \frac{\partial \lambda^{i}}{\partial x^{j}}-\frac{\delta_{i j}}{2 m} \frac{\partial \tau}{\partial t}\right]=0 \\
\left(p^{i}\right)^{2} p^{j}\left[-\frac{\delta_{i j}}{2 m^{2}} \frac{\partial \tau}{\partial x^{i}}\right]=0
\end{array}\right\} .
$$

\footnotetext{
${ }^{12}$ Please also note that the kinetic energy term of the Lagrangian is velocity dependent.
} 
The summation over $i$ and $j$ is implicit in the above set of equations. The above equations with the summation explicitly stated are written as

$$
\left\{\begin{array}{l}
{\left[-\frac{\partial \tau}{\partial t} U-\tau \frac{\partial U}{\partial t}-\frac{\partial U}{\partial x^{i}} \lambda^{i}-\frac{\partial f}{\partial t}\right]=0} \\
\sum_{i=1}^{3}\left(p^{i}\right)\left[\frac{\partial \lambda^{i}}{\partial t}-\frac{1}{m} \frac{\partial f}{\partial x^{i}}-\frac{1}{m} \frac{\partial \tau}{\partial x^{i}} U\right]=0 \\
\sum_{i=1}^{3} \sum_{j=1}^{3}\left(p^{i} p^{j}\right)\left[\frac{1}{m} \frac{\partial \lambda^{i}}{\partial x^{j}}-\frac{\delta_{i j}}{2 m} \frac{\partial \tau}{\partial t}\right]=0 \\
\sum_{i=1}^{3} \sum_{j=1}^{3}\left(p^{i}\right)^{2} p^{j}\left[-\frac{\delta_{i j}}{2 m^{2}} \frac{\partial \tau}{\partial x^{i}}\right]=0
\end{array}\right.
$$

Solving the last equation which is the only uncoupled equation reveals

$$
\therefore \tau=\tau(t)
$$

Furthermore, with the help of equation IV.31 and some regrouping, the remaining of the equations can be thought as a system of three equations and three unknowns. This set of equations is known as the Killing Equations:

$$
\text { Killing Equations } \equiv\left\{\begin{array}{l}
(K 1): \frac{\partial U}{\partial x^{i}} \lambda^{i}\left(t, x^{j}\right)+\frac{\partial}{\partial t}\left(U \tau(t)+f\left(t, x^{i}\right)\right)=0 \\
(K 2): \frac{\partial \lambda^{i}\left(t, x^{j}\right)}{\partial t}=\frac{1}{m} \frac{\partial f\left(t, x^{i}\right)}{\partial x^{i}} \\
(K 3): \frac{\partial \lambda^{i}\left(t, x^{j}\right)}{\partial x^{j}}=\frac{\delta_{i j}}{2} \dot{\tau}(t)+\alpha_{i j}
\end{array}\right\}
$$

where $\alpha_{i j}$ is a constant element of an antisymmetric matrix. The reason for the introduction of this constant term lies in the fact that in the third equation of IV.30, the product $p^{i} p^{j}$ is an element of a symmetric matrix for any $i$ and $j$ ranging from 1 to 3 . This matrix is in the form:

$$
s=\left[\begin{array}{lll}
p_{1} p_{1} & p_{1} p_{2} & p_{1} p_{3} \\
p_{2} p_{1} & p_{2} p_{2} & p_{2} p_{3} \\
p_{3} p_{1} & p_{3} p_{2} & p_{3} p_{3}
\end{array}\right]
$$

where the matrix is clearly symmetrical. Additionally, if

$$
\alpha_{i j}=\frac{1}{m} \frac{\partial \lambda^{i}}{\partial x^{j}}-\frac{\delta_{i j}}{2 m} \frac{\partial \tau}{\partial t}
$$


where $\alpha$ is an antisymmetric matrix, then equation 3 , in IV.30, becomes

$$
\sum_{i=1}^{3} \sum_{j=1}^{3} s_{i j} \alpha_{i j}=0
$$

which is always true because $S$ is a symmetric matrix whereas $\alpha$ is an antisymmetric matrix. Thus, by imposing that the term inside the bracket in equation 3 has to belong to an antisymmetric matrix, equation 3 will be satisfied globally for any value of $p^{i}$ and $p^{j}$, which is exactly what we seek after.

IV.31 is a set of three equations and three unknowns which are unfortunately coupled. Therefore, one has to find a way to uncouple the equations in order to solve them or reduce the three equations into one single equation. I shall choose the latter.

From K3,

$$
\frac{\partial \lambda^{i}\left(t, x^{j}\right)}{\partial x^{j}}=\frac{\delta_{i j}}{2} \dot{\tau}(t)+\alpha_{i j} .
$$

Integrating the above equation with respect to $x^{j}$ results in

$$
\lambda^{i}\left(t, x^{j}\right)=\frac{\delta_{i j}}{2} \dot{\tau}(t) x^{j}+\alpha_{i j} x^{j}+A^{i}(t)
$$

where $A^{i}(t)$ is a function of time that is the "constant" of integration when integrating K3. Furthermore, the product $\delta_{i j} x^{i}$ is equal to $x^{i}$ because $\delta_{i j}$ is the Kronecker delta, and this is exactly what Kroncker delta does. Thus, equation IV.32 can be simplified into

$$
\lambda^{i}\left(t, x^{j}\right)=\frac{1}{2} \dot{\tau}(t) x^{i}+\alpha_{i j} x^{j}+A^{i}(t)
$$

with the partial time derivative in the form

$$
\frac{\partial \lambda^{i}}{\partial t}=\frac{1}{2} \ddot{\tau} x^{i}+\dot{A}^{i} .
$$

From K2,

$$
\frac{\partial \lambda^{i}\left(t, x^{j}\right)}{\partial t}=\frac{1}{m} \frac{\partial f\left(t, x^{i}\right)}{\partial x^{i}}
$$

Using IV.33,

$$
\frac{1}{2} \ddot{\tau} x^{i}+\dot{A}^{i}=\frac{1}{m} \frac{\partial f\left(t, x^{i}\right)}{\partial x^{i}}
$$




$$
\therefore f\left(t, x^{i}\right)=\frac{m}{4} \ddot{\tau}\left(x^{i}\right)^{2}+\dot{A}^{i} x^{i}+B(t),
$$

where $B(t)$ is another "constant" of integration created in the process of integration with respect to $x^{i}$. Moreover, the partial time derivative of IV.34 can be calculated as

$$
\frac{\partial f\left(t, x^{i}\right)}{\partial t}=\frac{m}{4} \dddot{\tau}\left(x^{i}\right)^{2}+\ddot{A}^{i} x^{i}+\dot{B}(t) .
$$

From K1,

$$
\frac{\partial U}{\partial x^{i}} \lambda^{i}\left(t, x^{j}\right)+\frac{\partial}{\partial t}\left(U \tau(t)+f\left(t, x^{i}\right)\right)=0 .
$$

Using IV.35 and IV.32, the above equation can be evaluated further:

$$
\begin{gathered}
0=\frac{\partial U}{\partial x^{i}}\left(\frac{1}{2} \dot{\tau}(t) x^{i}+\alpha_{i j} x^{j}+A^{i}(t)\right)+\dot{\tau} U+\tau \frac{\partial U}{\partial t}+\frac{m}{4} \dddot{\tau}\left(x^{i}\right)^{2}+\ddot{A}^{i} x^{i}+\dot{B}(t) \\
=\left(\frac{m}{4}\left(x^{i}\right)^{2}\right) \dddot{\tau}+\left(\frac{1}{2} \frac{\partial U}{\partial x^{i}} x^{i}+U\right) \dot{\tau}+\left(\frac{\partial U}{\partial t}\right) \tau+\left(\frac{\partial U}{\partial x^{i}}\left(\alpha_{i j} x^{j}+A^{i}(t)\right)+\ddot{A}^{i} x^{i}+\dot{B}(t)\right) \\
\therefore\left(\frac{m}{4}\left(x^{i}\right)^{2}\right) \dddot{\tau}+\left(\frac{1}{2} \frac{\partial U}{\partial x^{i}} x^{i}+U\right) \dot{\tau}+\left(\frac{\partial U}{\partial t}\right) \tau+\left(\frac{\partial U}{\partial x^{i}} \alpha_{i j} x^{j}\right)+\left(\frac{\partial U}{\partial x^{i}} A^{i}(t)+\ddot{A}^{i} x^{i}+\dot{B}(t)\right)=0
\end{gathered}
$$

Finally, since the killing equations have been reduced to one single equation, and since this equation cannot be evaluated any further, the derivation of Noether's inverse theorem is complete.

What has been found is a set of equations (killing equations) that limits the choice for continuous transformations that could be used as the symmetrical transformations generating conserved quantities. In other words, not all continuous transformations are symmetrical; the symmetrical ones have to satisfy the killing equations or, equivalently, satisfy equation IV.36 (called the Auxiliary equation).

A remarkable feature of equation IV.36 is that "the function $\tau(t)=1$ is always a solution of the auxiliary equation for systems with time-independent Lagrangians. With this solution, the Noether's conserved charge coincides with the Hamiltonian $H$ " [10]. In order to see why this claim is true, one needs to substitute $U\left(x^{i}\right)$ for $U$ and $\tau=1$ for $\tau$ in equation IV.36. Doing so results in:

$$
\left(\frac{\partial U\left(x^{i}\right)}{\partial x^{i}} \alpha_{i j} x^{j}\right)+\left(\frac{\partial U}{\partial x^{i}} A^{i}(t)+\ddot{A}^{i} x^{i}+\dot{B}(t)\right)=0 .
$$

On one hand, from equation IV.32, 


$$
\lambda^{i}\left(t, x^{j}\right)=\frac{1}{2} \dot{\tau}(t) x^{i}+\alpha_{i j} x^{j}+A^{i}(t) a
$$

which has to be zero since only the time transformations are concerned. Setting $\lambda^{i}$ equal to zero requires

$$
A^{i}(t)=-\alpha_{i j} x^{j}=\text { const }
$$

which is due to the fact that $A^{i}(t)$ and $x^{j}$ are completely independent. Furthermore, putting IV.38 into IV.37 yields

$$
\begin{aligned}
0 & =\left(-\frac{\partial U\left(x^{i}\right)}{\partial x^{i}} A^{i}(t)\right)+\left(\frac{\partial U}{\partial x^{i}} A^{i}(t)+\ddot{A}^{i} x^{i}+\dot{B}(t)\right) \\
& =\dot{B}(t)
\end{aligned}
$$

On the other hand, from equation IV.34

$$
f\left(t, x^{i}\right)=\frac{m}{4} \ddot{\tau}\left(x^{i}\right)^{2}+\dot{A}^{i} x^{i}+B(t),
$$

which for $\tau=1$ and $A^{i}(t)=$ const yields

$$
f\left(t, x^{i}\right)=B(t)
$$

Looking back at equation IV.26, the function $f$ was introduced as $K / \varepsilon$ where

$$
\delta S=\int_{t_{1}}^{t_{2}}\left[\frac{d}{d t} K\left(x^{i}, t\right)\right] d t,
$$

based on the definition of invariance. In the case of time independent systems with time transformation of $\tau=1$, it is easy to see that the action does not change by a time transformation; thus, making $K(x, t)$, and in return $f$ (since $K / \varepsilon=f$ ), to be equal to a constant. If $f$ is a constant, then based on IV.40, $B(t)$ is a constant which makes equation IV.39 to always hold true. Therefore, for a conservative system, $\tau=1$ is always a solution to the auxiliary equation of IV.36. In addition, it is easy to see why the conserved quantity associated with $\tau=1$ for a conservative system is the Hamiltonian: based on equation IV.27, Noether's theorem states

$$
p^{i} \lambda^{i}-H \tau-f=\text { const. }
$$

In the above equation, $f$ is already found to be a constant, and $\lambda^{i}$ is zero due to the fact that only the time transformation of $\tau=1$ is considered. All these conditions would make

$$
H=\text { const }
$$

that is the Hamiltonian is conserved under $\tau=1$ transformation. 
As Noether's inverse theorem suggests, not all symmetrical transformations are necessarily constant transformations. According to equation IV.36, for a given potential energy and position vector, there could be symmetrical transformations that are none-linear and non-constant in nature. For example, for the one-dimensional harmonic oscillator with the Lagrangian

$$
L(x, t)=\frac{1}{2} p^{2}-\frac{1}{2} \omega_{0}^{2} x^{2},
$$

it can be shown that the auxiliary equation of IV.36 can be reduced to

$$
\dddot{\tau}(t)+4 \omega_{0}^{2} m \dot{\tau}(t)=0 .
$$

The general solution of this equation is in the form

$$
\tau(t)=a+b \cos \left(2 \omega_{0} t\right)+c \sin \left(2 \omega_{0} t\right)
$$

Where $a, b$, and $c$ are constants. As is apparent from the general solution, not only constant transformations are allowed, but also transformations in the form of sinusoidal functions could be allowed as well. Since both the constant and the sinusoidal transformations are symmetrical to the conservative mass-spring system (because they were derived from the auxiliary equations for this system), for each transformation, a unique conservation law will be obtained. This case will be studied in more detail in section V, Example 4.

\section{A Note on Noether's Inverse Theorem}

After deriving equation IV.36 mathematically and precisely, there is no question about the validity of the Noether's inverse theorem; however, the fact that it requires a third order differential equation to be solved for finding the symmetrical transformations makes it less useful as compared to the technique of guessing a transformation and seeing whether or not there exists a conservation charge for it. Thus, unless guessing technique does not lead to satisfactory results, the use of Noether's inverse theorem seems unjustified, especially for the conservative systems where guessing the symmetrical transformations is not a difficult task.

\section{NOETHER'S THEOREM THROUGH EXAMPLES}

The beauty of Noether's first theorem for classical dynamics is perhaps best understood through examples; therefore, in what follows, I shall present a few examples each of which offering a new perspective on understanding Noether's first theorem.

\section{Example 1 - Noether's Theorem and Conservation of Hamiltonian}

Consider a one dimensional system with the Lagrangian

$$
L=\frac{1}{2} m \dot{x}^{2}-U(x, t)
$$

in which only the time transforms (a constant transformation of time). This transformation of time can be mathematically expressed as 


$$
t^{\prime}=t+\delta t ; \delta t \equiv \varepsilon \tau(t)=\varepsilon,
$$

where $\tau(t)$ is set to 1 . Since only the time transformations are allowed,

$$
\delta x_{\mu} \equiv \varepsilon \lambda_{\mu}\left(t, x_{\mu}\right)=0,
$$

and thus the Noether's conserved charge equation (equation IV.22) reduces to:

$$
Q=\varepsilon H+K=\text { Const. }
$$

The Hamiltonian of a system is easy to calculate since the Lagrangian is known. Therefore, the only unknown that needs to be determined is $K$, which can be found using the definition of invariance given at the beginning of section IV (equation IV.8):

On one hand,

$$
L^{\prime}=L(x, \dot{x}, t+\varepsilon) \approx L+\frac{\partial L}{\partial t} \varepsilon
$$

On the other hand,

$$
\begin{gathered}
\int_{t_{1}}^{t_{2}} \frac{d}{d t}[K(x, t)] d t=\int_{t_{1}}^{t_{2}}{ }^{\prime} L^{\prime} d t-\int_{t_{1}}^{t_{2}} L d t \\
=\int_{t_{1}}^{t_{2}}\left[L^{\prime} \frac{d t}{d t}-L\right] d t
\end{gathered}
$$

in which a $U$-substitution was performed to put the two integrals inside one single integral with one single limit of integration. Now, based on V.1, $d t^{\prime}=d t$ which along with V.2 makes V.3 to become simplified further:

$$
\begin{aligned}
\int_{t_{1}}^{t_{2}}\left[\frac{d}{d t} K(x, t)\right] d t & =\int_{t_{1}}^{t_{2}}\left[L^{\prime}-L\right] d t \\
& =\int_{t_{1}}^{t_{2}}\left[\frac{\partial L}{\partial t} \varepsilon\right] d t \\
& =\int_{t_{1}}^{t_{2}}[-\dot{H} \varepsilon] d t \\
& =-H \varepsilon+\text { const. }
\end{aligned}
$$

Now that $K$ is known explicitly, it can be put into Noether's equation. This results in:

$$
H \varepsilon-H \varepsilon+\text { const }=\text { const }
$$

Or,

$$
\text { const }=\text { const }
$$


At first, this seems to be not all that powerful and wonderful as how I claimed the Noether's theorem is. However, the triviality (and of course the uselessness) of equation V.4 has nothing to do with the power of Noether's theorem itself. A non-symmetrical time transformation was selected; and as a result, a trivial statement was obtained, which of course is not a conservation law. However, if the physical system for which we assigned the Lagrangian

$$
L(x, \dot{x}, t)=\frac{1}{2} m \dot{x}^{2}-U(x, t)
$$

becomes a conservative system, meaning that there is no explicit time-dependence in the Lagrangian, then, a very different result, as compared to V.4, will be obtained. This case was studied at the end of section IV. Under the constant time transformation of $t^{\prime}=t+\varepsilon$ a conservative system conserves its Hamiltonian; unlike V.4, this is a proper conservation law! Restricting the system under analysis to a conservative system leads to the conclusion that, based on Noether's theorem, a constant time transformation is, in fact, a symmetrical transformation, which as expected, leads to a conservation law. More specifically, since the system is conservative, symmetry in time leads to conservation of total Energy. This statement is what Noether's theorem is mostly known for.

But, let us not forget about the most general form of a 1-D non-relativistic classical system, a system with the Lagrangian

$$
L(x, \dot{x}, t)=\frac{1}{2} m \dot{x}^{2}-U(x, t)
$$

Nothing appeared to be conserved under the constant transformation of time for this Lagrangian in general. As was mentioned above, nothing useful was obtained because $\tau=1$ is not a symmetrical transformation for a non-conservative system (even though it is for a conservative system!). Nevertheless, if one considers a different and none-constant time transformation, say

$$
t^{\prime}=t+\varepsilon(t+\text { const })
$$

one will end up with a valid conservation law for the system. The conservation law would be

$$
H\left(t+C_{1}\right)=C_{0}
$$

where $C_{0}$, and $C_{1}$ are constants ${ }^{13}$.

The main difference between a conservative system and a none-conservative system, from the point of view of the Noether's theorem, is that a conservative system is symmetrical under any continuous time transformation, and this transformation results in the conservation of total energy whereas a non-conservative system is symmetrical only under a selected group of continuous time transformation. In other words, Noether's theorem takes a strong and general form when it deals

\footnotetext{
13 The derivation of this conservation law is similar to the cases studied earlier: after a set of transportations is defined explicitly, one needs to find the explicit expression for $K$ using the definition of invariance. Once $K$ is determined, the Noether's conserve quantity can be easily found following equation IV.22.
} 
with a conservative system, but narrows its focus on only particular time transformations for nonconservative systems.

The only question that remains is whether or not any time transformation makes physical sense. I stated that if a non-conservative system undergoes transformation of the form

$$
t^{\prime}=t+\varepsilon(t+\text { const. })
$$

then a conservation law will be achieved. But, is such a transformation even possible? The answer is definitely yes, mathematically, but probably not physically. Unlike spacial coordinates, the time cannot be transformed artificially; thus, the only real time transformation is probably in the form of

$$
t^{\prime}=t+\varepsilon
$$

To the best knowledge of the author, that is the only time transformation that makes physical sense, at least in the realm of classical dynamics.

\section{Example 2 - Noether's Theorem and Conservation of Conjugate Momentum}

For the second example, imagine the same system, with the Lagrangian

$$
L=\frac{1}{2} m \dot{x}^{2}-U(x, t)
$$

in which only the spacial coordinates transform rather than the time. This transformation of the space (one dimensional for the sake of simplicity) can be mathematically expressed as

$$
x^{\prime}=x+\delta x
$$

where $x^{\prime}$ is the very next position in space after $x$ and $\delta x \equiv \varepsilon \lambda(x, t)=\varepsilon$. Since only the space transforms, $\delta t$ is equal to zero and thus the Noether's conserved charge becomes:

$$
p \delta x-K=\text { Const }
$$

The conjugate momentum of a system, $p$, is always easy to calculate when the Lagrangian is known. Therefore, similar to the previous example, the only unknown that needs to be determined is $K$, which can be found using equation V.3 and noting that

$$
L^{\prime}=L(x+\varepsilon, \dot{x}, t) \approx L+\frac{\partial L}{\partial x} \varepsilon
$$

Putting V.5 into V.3 results in

$$
\begin{aligned}
\int_{t_{1}}^{t_{2}}\left[\frac{d}{d t} K(x, t)\right] d t & =\int_{t_{1}}^{t_{2}}\left[L^{\prime} \frac{d t^{\prime}}{d t}-L\right] d t \\
& =\int_{t_{1}}^{t_{2}}\left[L^{\prime}-L\right] d t
\end{aligned}
$$




$$
\begin{aligned}
& =\int_{t_{1}}^{t_{2}}\left[\frac{\partial L}{\partial x} \varepsilon\right] d t \\
& =\int_{t_{1}}^{t_{2}}[\dot{p} \varepsilon] d t \\
& =p \varepsilon+\text { const } .
\end{aligned}
$$

Knowing the exact expression for $K$, one can substitute this expression back into equation V.5:

$$
p \varepsilon-p \varepsilon+\text { const }=\text { const. }
$$

Or,

$$
\text { const }=\text { const. }
$$

In other words, similar to the time transformation example for the non-conservative systems, there is no conservation law that can be resulted from the choice of a non-symmetrical transformation. However, what if the Lagrangian of this example was different? What if the considered system is not under the influence of any external force (i.e. $U(x, t)=0)$ ? In this case, $K$ can be calculated as

$$
\begin{aligned}
\int_{t_{1}}^{t_{2}}\left[\frac{d}{d t} K(x, t)\right] d t & =\int_{t_{1}}^{t_{2}}\left[L^{\prime} \frac{d t^{\prime}}{d t}-L\right] d t \\
& =\int_{t_{1}}^{t_{2}}\left[L^{\prime}-L\right] d t \\
& =\int_{t_{1}}^{t_{2}}\left[\frac{\partial L}{\partial x} \varepsilon\right] d t \\
& =\int_{t_{1}}^{t_{2}}[0 \times \varepsilon] d t \\
& =\text { const }
\end{aligned}
$$

which when used in Noether's conserved charge equation, it yields a conservation law:

$$
\text { pe }- \text { const }=\text { const. }
$$

Or,

$$
p=\text { const }
$$

Now, as expected, there is a proper conservation law for this system: the conservation law is the conservation of the momentum conjugate to the spacial variable that transforms in space. In other words, the symmetry in space leads to the conservation of momentum, of course, in the case of absence of external forces. This is another profound statement about Nature suggested by the Noether's first theorem. Since the derivation included the conjugate momentum rather than the kinetic momentum, one can easily think about $x$ as the angular position, replace the mass term in the Lagrangian with the moment of inertia, and consider $p$ as the angular momentum. Then, another profound statement can be made with the help of Noether's first theorem: the symmetry in orientation of space leads to conservation of angular momentum (in the absence of external torques of course). 
Nonetheless, similar to the previous example, there exist some spacial transformations that make a system under the influence of a potential energy to have a conserved quantity which can be expressed in terms of conjugate momentum. Thus, again, Noether's theorem does not state that conserved quantities do not exist for a system with a non-zero potential, but rather Noether theorem requires certain forms of spacial transformations to occur in order for a conserved quantity to exist.

Now, the only question that remains is whether or not any spacial transformation is even physically possible. To the knowledge of the author, the answer to this question, unlike the case of time transformation, is yes.

\section{Example 3 - Noether's Theorem and Transformation of all Coordinates}

Again, let us consider a general classical dynamic Lagrangian of the form

$$
L=\frac{1}{2} m \dot{x}^{2}-U(x, t) .
$$

Following the exact same logic as of the previous examples, for a continuous time transformation of the form $t^{\prime}=t+\delta t$ and for a continuous space transformation of the form $x^{\prime}=x+\delta x$, the only factor inside Noether's conserved charge equation that is truly unknown is $K$. For the case in hand, $K$ can be determined as follows.

The transformations are

$$
\begin{aligned}
& x^{\prime}=x+\delta x=x+\varepsilon \lambda(x, t) \\
& t^{\prime}=t+\delta t=t+\varepsilon \tau(t) \\
& \dot{x}^{\prime}=\dot{x}+\delta v=\dot{x}+\varepsilon(\dot{\lambda}-\dot{x} \dot{\tau})
\end{aligned}
$$

To first order approximation,

$$
L^{\prime}=L(x+\delta x, t+\delta t, \dot{x}+\delta v) \approx L+\left(m \dot{x} \delta v-\frac{\partial U}{\partial x} \delta x-\frac{\partial U}{\partial t} \delta t\right) .
$$

Using the definition of invariance, and knowing that $d t^{\prime} / d t=1+\varepsilon \dot{\tau}$,

$$
\begin{aligned}
\int_{t_{1}}^{t_{2}}\left[\frac{d}{d t} K(x, t)\right] d t & =\int_{t_{1}}^{t_{2}}\left[L^{\prime} \frac{d t^{\prime}}{d t}-L\right] d t \\
& =\int_{t_{1}}^{t_{2}}\left[L^{\prime}(1+\varepsilon \dot{\tau})-L\right] d t \\
& =\int_{t_{1}}^{t_{2}}\left[\left(L+\frac{\partial L}{\partial t} \delta t+\frac{\partial L}{\partial x} \delta x+\frac{\partial L}{\partial \dot{x}} \delta v\right)(1+\varepsilon \dot{\tau})-L\right] d t \\
K & =\int\left[\left(L+\frac{\partial L}{\partial t} \delta t+\frac{\partial L}{\partial x} \delta x+\frac{\partial L}{\partial \dot{x}} \delta v\right)(\varepsilon \dot{\tau})+\left(\frac{\partial L}{\partial t} \delta t+\frac{\partial L}{\partial x} \delta x+\frac{\partial L}{\partial \dot{x}} \delta v\right)\right] d t \\
& =\int[(L-\dot{H} \delta t+\dot{p} \delta x+p \delta v)(\varepsilon \dot{\tau})+(-\dot{H} \delta t+\dot{p} \delta x+p \delta v)] d t
\end{aligned}
$$




$$
=\int\left[\left(\frac{1}{2} m \dot{x}^{2}-U(x, t)-\frac{\partial U}{\partial t} \delta t+m \ddot{x} \delta x+m \dot{x} \delta v\right)(\varepsilon \dot{\tau})+\left(-\frac{\partial U}{d t} \delta t+m \ddot{x} \delta x+m \dot{x} \delta v\right)\right] d t
$$

where

$$
\begin{aligned}
& \delta t=\varepsilon \tau(t) \\
& \delta x=\varepsilon \lambda(x, t) \\
& \delta v=\varepsilon(\dot{\lambda}-\dot{x} \dot{\tau}) .
\end{aligned}
$$

Substituting the above back into Noether's conserved charge equation yields

$$
Q=p \delta x-H \delta t-\int\left[\left(\frac{1}{2} m \dot{x}^{2}-U(x, t)-\frac{\partial U}{\partial t} \delta t+m \ddot{x} \delta x+m \dot{x} \delta v\right)(\varepsilon \dot{\tau})-\frac{\partial U}{\partial t} \delta t+m \ddot{x} \delta x+m \dot{x} \delta v\right] d t
$$

The above equation is perhaps the most general conservation law for a system defined in the framework of classical particle dynamics. Knowing the transformations explicitly in addition to knowing the explicit expressions of potential and kinetic energy would allow one to determine the conserved charge associated with the chosen transformation (if there is any).

The cases where either the time or the spacial coordinates transforms continuously by a small amount $\varepsilon$ were discussed earlier in previous examples. Now, let us examine the case where both are in effect. Since both the time and the space transforms constantly by $\varepsilon, \delta v$ will be equal to zero, according to equation V.7, Thus, the calculated general form of $K$ in equation V.8 for the Noether's conserved charge will become

$$
\begin{aligned}
Q & =m \dot{x} \varepsilon-\left(\frac{1}{2 m} p^{2}+U(x, t)\right) \varepsilon-\int\left(\frac{d}{d t}(\varepsilon m \dot{x})-\frac{d}{d t}\left(\varepsilon\left(\frac{1}{2 m} p^{2}+U(x, t)\right)\right)\right) d t \\
& =(m \dot{x} \varepsilon-\varepsilon m \dot{x})+\left(-\varepsilon\left(\frac{1}{2 m} p^{2}+U(x, t)\right)+\varepsilon\left(\frac{1}{2 m} p^{2}+U(x, t)\right)\right) \\
& =0
\end{aligned}
$$

or no conservation law at all ${ }^{14}$. This, once again, highlights the fact that the simple transformations would not work for the non-conservative systems. The reason that the inverse Noether's theorem was provided is to address this issue. Non-conservative systems require more complex transformations to reveal their symmetries.

\section{Example 4 - The 1-D Conservative Harmonic Oscillator}

\footnotetext{
${ }^{14}$ Please note that the identity $\partial U / \partial d t=-\partial L / \partial t=\dot{H}$ was used to simplify the expression for $Q$.
} 
Let us approach this example differently. Instead of imposing the form of time or space transformations prior to solving for the conserved charges (i.e. using Noether's theorem), let us first use Noether's inverse theorem to look for a family or families of symmetrical transformations and then, find their corresponding conserved charges.

Recalling the Noether's inverse theorem, the following equation has to hold true for all the symmetrical time transformations:

$$
\left(\frac{m}{4}\left(x^{i}\right)^{2}\right) \dddot{\tau}+\left(\frac{1}{2} \frac{\partial U}{\partial x^{i}} x^{i}+U\right) \dot{\tau}+\left(\frac{\partial U}{\partial t}\right) \tau+\left(\frac{\partial U}{\partial x^{i}} \alpha_{i j} x^{j}\right)+\left(\frac{\partial U}{\partial x^{i}} A^{i}(t)+\ddot{A}^{i} x^{i}+\dot{B}(t)\right)=0
$$

where $A^{i}(t)$ is a function of time appearing in the most general form of an acceptable symmetrical spacial transformation, equation IV.32. In order to make the solving process of the above equation easier, only one dimension and a conservative mass-spring system (with mass of $1 \mathrm{~kg}$ ) could be considered. These restrictions would result in

$$
\alpha_{i j}=\alpha_{11}=0
$$

(since $\alpha_{i j}$ is an element of an antisymmetric matrix) and

$$
\frac{\partial U}{\partial t}=0
$$

Thus, the auxiliary equation can be reduced to

$$
\left[\left(\frac{1}{4}(x)^{2}\right) \dddot{\tau}+\left(\frac{1}{2} \frac{\partial U}{\partial x^{i}} x^{i}+U\right) \dot{\tau}\right]+\left[\left(\frac{\partial U}{\partial x^{i}} A^{i}(t)+\ddot{A}^{i} x^{i}+\dot{B}(t)\right)\right]=0 .
$$

The two expressions in each bracket would result in a different conserved charge [9]. Looking at the first bracket only, according to Noether's inverse theorem, the solution to the following equation would yield a conservation law [10]:

$$
\left[\left(\frac{1}{4}(x)^{2}\right) \dddot{\tau}+\left(\frac{1}{2} \frac{d U}{d x} x+U\right) \dot{\tau}\right]=0
$$

where

$$
U=U(x)=\frac{1}{2} \omega_{0}^{2} x^{2}
$$

Putting V.10 into V.9 results in

$$
\begin{aligned}
& \frac{1}{4} \dddot{\tau}+\omega_{0}^{2} \dot{\tau}=0 \\
& \therefore \tau(t)=C_{1} \sin \left(2 \omega_{0} t\right)+C_{2} \cos \left(2 \omega_{0} t\right)+C_{3}
\end{aligned}
$$


where $C_{1}, C_{2}$, and $C_{3}$ are constant. Therefore, for a conserved one dimensional harmonic oscillator with mass of $1 \mathrm{~kg}$, any time transformation which belongs to the family of transformations of

$$
\tau(t)=C_{1} \sin \left(2 \omega_{0} t\right)+C_{2} \cos \left(2 \omega_{0} t\right)+C_{3}
$$

is guaranteed to result in a conserved charge.

The trivial case of $\tau(t)=C_{3}$ results in the conservation of total energy which was proven in the previous sections for the general case of the conservative systems. Nevertheless, there are two more conserved quantities which are obtained by setting $C_{3}$ and in either of $C_{2}$ and $C_{1}$ equal to zero:

$$
\begin{aligned}
& \text { For } C_{3}=C_{2}=0: Q_{2}=\frac{1}{2}\left(p^{2}-\omega_{0}^{2} x^{2}\right) \cos \left(2 \omega_{0} t\right)+\omega_{0} p x \sin \left(2 \omega_{0} t\right) \\
& \text { For } C_{3}=C_{1}=0: Q_{3}=\frac{1}{2}\left(p^{2}-\omega_{0}^{2} x^{2}\right) \sin \left(2 \omega_{0} t\right)+\omega_{0} p x \cos \left(2 \omega_{0} t\right)
\end{aligned}
$$

If there is any doubt about the validity of the obtained conservation laws, one could simply substitute the exact expressions of momentum and position of a mass in a mass-spring system (mass of $1 \mathrm{~kg}$ ) into the above two equations to observe that the conservation laws are in fact correct! "The above two conserved charges are related to the time evolution of both the kinetic energy and the potential energy" [10]. The above quantities also display the power of Noether's equation: without the Noether's formalism, none of the above conservation laws could be possibly obtained.

\section{Example 5 - An Odd Case: The Lane-Emden Equation}

This example is very different from any other previously mentioned example since it is not in the framework of classical particle dynamics; yet, we can treat it as if it is, thanks to the elegance of Noether's theorem.

"Polytropes are self-gravitating gaseous spheres that were, and still are, very useful as a crude approximation to more realistic stellar models" [11]. They are described by a family of equations of state in hydrostatic equilibrium ${ }^{15}$. The equations of state take the assumption that

$$
P=k \rho^{\gamma}
$$

where $P$ is the pressure, $k$ and $\gamma$ are constants, and $\rho$ is the density of gas. Combining the hydrostatic equilibrium equation with V.13 would lead to what is known as the Lane-Emden Equation, which takes the following form [11]:

\footnotetext{
15 "For the majority of the life of a star, the gravitational force (due to the mass of the star) and the gas pressure (due to energy generation in the core of the star) balance, and the star is said to be in 'hydrostatic equilibrium"[12].
} 


$$
\frac{1}{\xi^{2}} \frac{d}{d \xi}\left(\xi^{2} \frac{d \theta}{d \xi}\right)=-\theta^{n}
$$

where,

$$
\begin{gathered}
\xi \equiv \frac{r}{\left(\sqrt{\frac{n+1}{4 \pi G} k \lambda^{\frac{1-n}{n}}}\right)} \\
\rho \equiv \lambda \theta^{n} \\
\gamma \equiv \frac{n+1}{n}
\end{gathered}
$$

for an integer $n$, a radial distance $r$ from the center of the gaseous sphere, polytropic temperature $\theta$, and central density $\lambda[11]$.

The lane-Emden equation, in actuality, is a family of equations because of the existence of the integer $n$. To make the calculations easier let us only analyze the case when the integer $n$ equals to 5. Moreover, let us use a more familiar set of symbols instead of the standard astrophysical symbols used in the Lane-Emden equation. Using $t$ instead of $\xi, x$ instead of $\theta$ and setting $n=5$ results in:

$$
\begin{aligned}
& \frac{1}{t^{2}} \frac{d}{d t}\left(t^{2} \frac{d x}{d t}\right)=-x \\
& \ddot{x}=-x^{5}-\frac{2}{t} \dot{x} .
\end{aligned}
$$

Note that $t$ is NOT time and $x$ is NOT position. Now, let us construct a Lagrangian based on $\mathrm{V} .15$ even though it will not have any physical meaning due to $t$ and $x$ not having their usual meanings.

Starting from

$$
\ddot{x}=-x^{5}-\frac{2}{t} \dot{x}
$$

And multiplying both sides of the equation by $t^{2}$ results in:

$$
t^{2} \ddot{x}=-t^{2} x^{5}-2 t \dot{x}
$$

or,

$$
\frac{d}{d t}\left(t^{2} \dot{x}\right)=-t^{2} x^{5}
$$

equivalently. Furthermore, comparing V.16 with the Euler-Lagrange equations leads to 


$$
\left\{\begin{array}{l}
\frac{\partial L}{\partial x}=-t^{2} x^{5} \\
\frac{\partial L}{\partial \dot{x}}=t^{2} \dot{x}
\end{array}\right\} \Rightarrow\left\{\begin{array}{l}
L=-\frac{1}{6} t^{2} x^{6}+A(t, \dot{x}) \\
L=\frac{1}{2} t \dot{x}^{2}+B(x, t)
\end{array}\right\}
$$

Where $A(t, \dot{x})$ and $B(x, t)$ are integration "constants". Assuming

$$
A(t, \dot{x})=\frac{1}{2} t^{2} \dot{x}
$$

and

$$
B(x, t)=-\frac{1}{6} t^{2} x^{6}
$$

The Lagrangian can be written as

$$
L(t, x, \dot{x})=t^{2}\left(\frac{\dot{x}^{2}}{2}-\frac{x^{6}}{6}\right)
$$

which of course is not unique (one could pick other expressions for $A(t, \dot{x})$ and $B(t, x)$ ). Now, the astrophysical system of a gaseous sphere is ready to be put into Noether's formalism. Since, the Lagrangian of the system is clearly a time-dependent one, we already have seen that the normal transformations of the form $t+\varepsilon$ or $x+\varepsilon$ would not lead to a conservation law. Either Noether's inverse theorem has to be invoked or an educated guess needs to be made in order to take advantage of Noether's theorem fully (i.e. finding a non-zero conserved charge). Since invoking Noether's inverse theorem would require solving a third order differential equation, making an educated guess is a better choice.

It is one of the characteristics of the Lane-Emden equation that if $x(t)$ is a solution of the Lane-Emden equation, then a rescaled version of $x(t)$ in the form of $e^{\varepsilon} x\left(e^{\varepsilon} t\right)$ is also a solution [13]. Therefore, it is an educated guess to try a transformation in the form of $e^{\varepsilon} x\left(e^{\varepsilon} t\right)$.

If

$$
x^{\prime}=e^{\varepsilon} x\left(e^{\varepsilon} t\right)
$$

then, up to the first order approximation,

$$
\begin{aligned}
& x^{\prime}=(1+\varepsilon)(x+\varepsilon \dot{x} t) \\
& \therefore x^{\prime}=x+\varepsilon(x+\dot{x} t) .
\end{aligned}
$$

Thus, one complete set of transformation can be explicitly written as

$$
\begin{aligned}
t^{\prime} & =t \\
x^{\prime} & =x+\varepsilon(x+\dot{x} t) \\
\dot{x}^{\prime} & =\dot{x}+\varepsilon(2 \dot{x}+\ddot{x} t) .
\end{aligned}
$$


As a result, the Noether's conserved charge takes the form

$$
Q=\frac{\partial L}{\partial \dot{x}}(\varepsilon(x+\dot{x} t))-K
$$

where

$$
p=\frac{\partial L}{\partial \dot{x}}=t^{2} \dot{x}
$$

Similar to the previous examples, $K$ is the real unknown of the Noether's equation which needs to be determined:

On one hand,

$$
\begin{aligned}
\int_{t_{1}}^{t_{2}}\left[\frac{d}{d t} K(x, t)\right] d t & =\int_{t_{1}}^{t_{2}}\left[L^{\prime} \frac{d t^{\prime}}{d t}-L\right] d t \\
& =\int_{t_{1}}^{t_{2}}\left[L^{\prime}-L\right] d t .
\end{aligned}
$$

On the other hand,

$$
\begin{aligned}
& L^{\prime}=L(t, x+\delta x, \dot{x}+\delta \dot{x}) \\
& \Rightarrow L^{\prime}-L \approx \frac{\partial L}{\partial x} \delta x+\frac{\partial L}{\partial \dot{x}} \delta \dot{x}
\end{aligned}
$$

Evaluating V.19 using V.17 and putting V.19 into V.18 yields

$$
K=\int t^{2}\left(\dot{x} \delta \dot{x}-x^{5} \delta x\right) d t
$$

At this stage of calculation, the explicit time-dependent forms of $x$ and its first and second total derivatives have to be known in order to proceed further. For the particular Lane-Emden equation that we are considering (i.e. $\mathrm{n}=5), x$ accepts the form [11]

$$
x(t)=\left(1+\frac{t^{2}}{3}\right)^{-1 / 2} .
$$

Now, the function $K$ of Noether's conserved charge can be determined using $x$ and its first and second total derivatives. The result is

$$
K=-\frac{9 t^{3}}{\left(t^{2}+3\right)^{3}} \varepsilon
$$

Putting this $K$ into Noether's equation results in

$$
Q=t^{2} \dot{x}(\varepsilon(x+\dot{x} t))+\frac{9 t^{3}}{\left(t^{2}+3\right)^{3}} \varepsilon
$$


or equivalently (after some simplification and reusing the standard astrophysical notation):

$$
\xi^{2}\left(\xi\left(\frac{d \theta}{d \xi}\right)^{2}+\theta \frac{d \theta}{d \xi}+\frac{1}{3} \xi \theta^{6}\right)=\text { const } \text {. }
$$

which is a non-zero conserved quantity. Although the exact meaning of the obtained conservation law is not obvious, its validity is undeniable. This is the power of Noether's theorem; it allows us to find conservation laws for systems, such as of the one described by the Lane-Emden equation, that one (such as the author) does not have much information about. This is remarkable!

\section{CONCLUSION}

In this study, continuous symmetries and their relation to conserved quantities have been discussed in great detail through the use of Noether's first theorem as well as Noether's inverse theorem in the framework of classical dynamics. Noether's theorem states that symmetries of Nature are tied to the conservation laws: symmetry in time leads to conservation of energy, symmetry in space leads to conservation of momentum, and symmetry in spacial orientation leads to the conservation of angular momentum.

Although the mentioned statements are what Noether's theorem is mostly known for, if one studies Noether's theorem in great depth alongside the lesser known Noether's inverse theorem, one will observe that there is much more to the connection between continuous symmetries and conserved quantities that the mentioned three statements about conservation of energy, momentum, and angular momentum. After finishing this study, I have the following remarks about Noether's theorem and continuous symmetries in general:

1. With the help of Noether's inverse theorem (the fact that the solution to equation IV.36 exists as long as the position vector and potential energy along with their partial derivatives are continuous along $t$ [9]) it can be concluded that, for any given system, whether timedependent or time-independent, there is a set of symmetrical transformations that would make that system to conserve a quantity. That quantity could be as well understood as the total energy or a lesser known one such as $Q_{1}$ in equation V.11 or $Q$ in equation V.21.

2. Continuous transformations that are symmetrical for a given system may not be symmetrical for another system; thus, the notion of symmetry is relative.

3. Noether' theorem and the mathematical relation that exists between continuous symmetries and conservation laws are so powerful that they could be used to find conservation laws for systems that are not in the framework of classical dynamics as well, without knowing much about the system except a second order differential equation. The systems described by the Lane-Emden equation are among such systems.

4. It was shown that for each symmetrical transformation, there exists a conservation law. Although any solution to equation IV.36 is a symmetrical transformation and is mathematically possible, some solutions of IV.36 seem to be not physically possible despite the fact that they give valid (and observable) conservational laws. For example, a sinusoidal transformation of time is mathematically possible and will give valid 
conservation laws, but, is such transformation of time possible? Since the conservation law given by a sinusoidal transformation of time is valid, does that mean that such transformation takes place in actuality? I have been unable to find convincing answers to the stated two questions. 


\section{REFERENCES}

[1] L. M. Lederman and C.T. Hill, Symmetry and the Beautiful Universe. Amherst, N.Y., USA : Prometheus Books, 2004.

[2] R. P. Feynman, R. B. Leighton, and M.L. Sands, The Feynman Lectures on Physics, Volume I. CA, USA: California Institute of Technology, 2013. [Online]. Available:

http://www.feynmanlectures.caltech.edu/I_toc.html

[3] "Pseudovector", Wolfram MathWorld. April 16, 2018. [Online]. Available:

http://mathworld.wolfram.com/Pseudovector.html

[4] R. A. Serway, C. J. Moses, and C. A. Moyer, "Quantum mechanics in three dimensions" in Modern Physics, 3rd ed. Belmont, CA, USA : Thomson Brooks/Cole, 2005, pp. 260-294

[5] "Gauge transformation", Encyclopedia of Mathematics, April 16, 2018. [Online]. Available: https://www.encyclopediaofmath.org/index.php/Gauge transformation

[6] C. G. Gray and E. F. Taylor, "When action is not least," AAPT, vol. 75, iss. 5. , pp 434-458, Apr. 2007. [Online]. doi: $10.1119 / 1.2710480$

[7] D. E. Neuenschwander, Emmy Noether's Wonderful Theorem. Baltimore, Md, USA: Johns Hopkins University Press, 2011

[8] M. Bañados and I. A. Reyes, "A short review on Noether's theorems, gauge symmetries and boundary terms," arXiv. Pp. 1-75, Aug. 2017. [Online]. doi: 10.1142/S0218271816300214

[9] J. Struckmeier and C. Riedel, "Noether's theorem and Lie symmetries for time-dependent Hamilton-Lagrange systems," APS, vol. 66, iss. 6. , pp 1-12, Dec. 2002. [Online]. doi: 10.1103/PhysRevE.66.066605

[10] J. Struckmeier, "Noether's theorem and invariants for time-dependent Hamilton-Lagrange systems", Frankfurt Institute for Advanced Studies, Jan 10, 2002. [Online]. Available:

https://fias.uni-frankfurt.de/ struckmeier/hp/thsem/invarcoll.pdf

[11] "Polytropes," Princeton University, Department of Astrophysical Science. April 28, 2018. [Online]. Available: https://www.astro.princeton.edu/ gk/A403/polytrop.pdf

[12] "Hydrostatic Equilibrium," Swinburne University of Technology. April 28, 2018. [Online]. Available: http://astronomy.swin.edu.au/cosmos/H/Hydrostatic+Equilibrium

[13] G. Gorni and G. Zampieri, "Revisiting Noether's theorem on constants of motion," $J$. Nonlinear Math. Phys, vol. 21, iss. 1, pp. 43-73, Feb 18, 2014. [Online]. doi:

10.1080/14029251.2014.894720 